\title{
Electromagnetic Scattering Coefficients for Concen- tric Spheres and the Problem of Interference Free Enclosures
}

\author{
R. A. Eldred, H. A. Lasitter, and J. Roberts \\ Contribution From the U.S. Naval Civil Engineering Laboratory, Port Hueneme, Calif.
}

(Received January 23, 1964; revised April 20, 1964)

\begin{abstract}
With the purpose of increasing the information about the lower frequency shielding effectiveness of closed structures composed of various arbitrary materials, an idealized problem is considered of the scattering and absorption of a plane electromagnetic wave impinging on a spherical shell. The region between the outer and inner radius of the sphere contains an arbitrary material; the other two regions are free space. The absorption and scattering coefficients for the incident plane wave are expressed as an infinite sum of spherical waves, using spherical Bessel functions. The coefficients are calculated numerically and shown for a large range of complex material parameters and frequencies from $100 \mathrm{kc} / \mathrm{s}$ to $1000 \mathrm{Mc} / \mathrm{s}$. Equations were programmed in FORTRAN, and numerical calculations accomplished on the IBM 1620 computer. Combinations of complex material properties of permeability, permittivity, and conductivity were sought which gave large, broadband absorption coefficients. Several promising compositions have resulted, and are presented. The effects of varying the individual parameters are also discussed.
\end{abstract}

\section{Introduction}

In order to utilize a mathematically complete and general solution to the boundary value problem, the symmetrical configuration of a spherical shell has been used to approximate the closed walls of the normally cubical or rectangular shielded enclosure. The behavior of the impinging plane wave electromagnetic field external to the spherical structure is considered. In order to completely describe the perturbing effect of the spherical shell, knowledge of the field outside, inside, and within the shell material is necessary. This investigation has been primarily directed toward a systematic exploratory search for wall (or shell, in this case) materials that will give uniform absorption of plane EM waves in the broad spectrum between 0.1 and $1000 \mathrm{Mc} / \mathrm{s}$.

The assumptions made in this investigation are:

1. The spherical structure is isolated in space,

2. the wave incident on the surface of the outer sphere is a plane wave,

3. the shell material is homogeneous and isotropic,

4. the shell is composed of an arbitrary material with complex properties of $\mu^{*}, \epsilon^{*}$, and $\sigma$. The inner sphere and the exterior region is free space.

The geometry of the spherical configuration is shown in figure 1. For this case, the equations describing the absorption $\left(C_{a}\right)$ and scattering $\left(C_{s}\right)$ coefficients have been derived (appendix A). The Mie scattering equations [Stratton, 1941] expanded to two concentric spheres by Salati [1962], are essentially those used in this paper. We have found that it is possible to extend the equations to cover three concentric spheres but only the two sphere solution is presented here. A general solution for any number of concentric spheres has been given by Wait [1962 and 1964].

The shell material parameters have been systematically varied over a wide range of values. Ranges of values of parameters were selected generally on the basis of known conductors or nonconductors. The case of high conductors yields considerable simplification of the $C_{s}$ and $C_{a}$ equations and has been treated separately. The dimensional properties of the spherical shell structure have, in most cases, been selected to approximate the dimensions of actual 


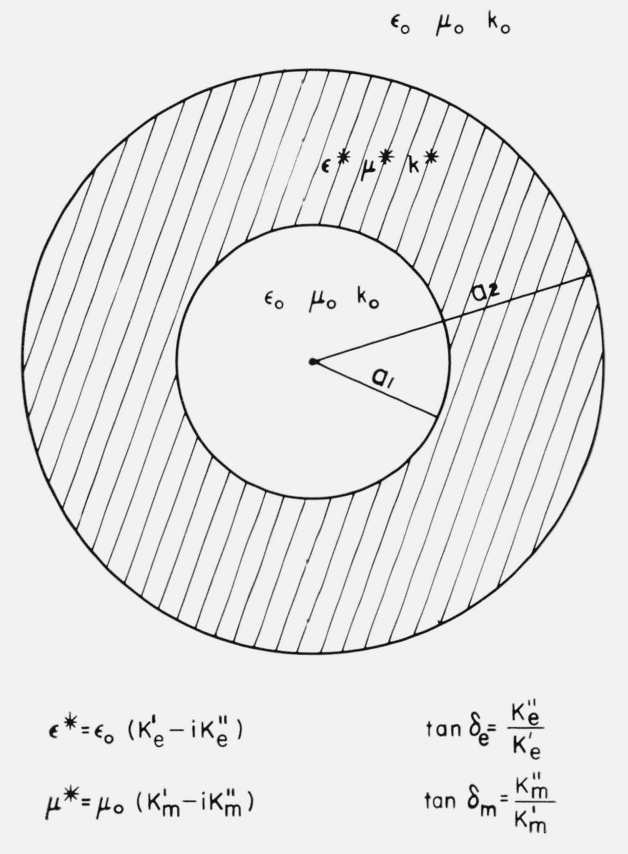

Figure 1. Diagram of spherical configuration. Inner and outer regions are free spare.

shielded enclosures. The majority of data was computed on the basis of $a_{2}$ (outer radius) $=5$ $\mathrm{m}(16.4 \mathrm{ft})$ and a shell thickness $\left(a_{2}-a_{1}\right)=0.15 \mathrm{~m}(5.9 \mathrm{in}$.$) . In many cases data were taken at$ different radii to show the variation in $C_{a}$ and $C_{s}$. The effect of shell thickness variation was also considered.

Approximately 150 separate combinations of material parameters or dimensional variations were computed. Only a summary of the materials that gave large absolute values of absorption coefficients and broadband absorption is given here.

\section{Absorption and Scattering Coefficients for the Case of Arbitrary Materials}

The absorption coefficient, $C_{a}$, is the ratio of the absolute absorption cross section to the geometrical cross section. It is also the ratio of the total power absorbed to the power of the incident wave crossing an area equal to the geometric cross section. Similar definitions hold for the scattering coefficient, $C_{s}$.

According to (A15) and (A16) in the appendix, the scattering and absorption coefficients may be expressed in terms of the external field coefficients, $a_{n}^{r}$ and $b_{n}^{r}$, as

$$
\begin{aligned}
& C_{s}=\frac{2}{\xi_{2}^{2}} \sum_{n=1}^{\infty}(2 n+1)\left(\left|a_{n}^{r}\right|^{2}+\left|b_{n}^{r}\right|^{2}\right) \\
& C_{a}=-\frac{2}{\xi_{2}^{2}} \sum_{n=1}^{\infty}(2 n+1)\left(\left|a_{n}^{r}\right|^{2}+\left|b_{n}^{r}\right|^{2}+\operatorname{Re} a_{n}^{r}+\operatorname{Re} b_{n}^{r}\right) .
\end{aligned}
$$

The field coefficients may be expressed in terms of first and second spherical Hankel functions, $h_{n}^{(1)}$ and $h_{n}^{(2)}$ and spherical Bessel functions of the first kind, $j_{n}$. The two external coefficients have identical form except for the interchange of the relative admittance of the material $\left(g=\sqrt{K_{e} / K_{m}}\right)$ and the relative impedance $\left(Z=\frac{1}{g}=\sqrt{K_{m} K_{e}}\right)$. Writing only one of these coefficients, we have from (A7)

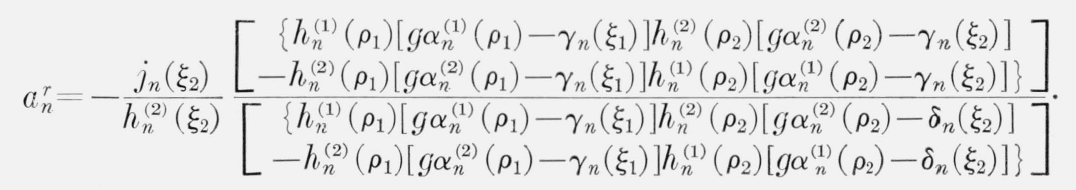


where

and

$$
\xi_{1}=k_{0} a_{1}, \xi_{2}=k_{0} a_{2}, \rho_{1}=k a_{1}, \rho_{2}=k a, \quad\left(k=k_{0} \sqrt{K_{m} K_{e}}\right)
$$

$$
\begin{gathered}
\alpha_{n}^{(l)}(x)=\frac{h_{n-1}^{(l)}(x)}{h_{n}^{(l)}(x)}-\frac{n}{x} \\
\gamma_{n}(x)=\frac{j_{n-1}(x)}{j_{n}(x)}-\frac{n}{x} \\
\delta_{n}(x)=\frac{h_{n-1}^{(2)}(x)}{h_{n}^{(2)}(x)}-\frac{n}{x}
\end{gathered}
$$

The material is characterized by complex permeability and permittivity,

where

$$
\begin{aligned}
& \epsilon^{*}=\epsilon_{0}\left(K_{e}^{\prime}-i K_{e}^{\prime \prime}\right)=\epsilon_{0} K_{e} \\
& \mu^{*}=\mu_{0}\left(K_{m}^{\prime}-i K_{m}^{\prime \prime}\right)=\mu_{0} K_{m}
\end{aligned}
$$

$$
\begin{aligned}
& K_{e}^{\prime \prime}=K_{e}^{\prime} \text { loss tangent for dielectrics } \\
& K_{e}^{\prime \prime}=\frac{\mu_{0} c^{2} \sigma}{\omega} \quad \text { for conductors. }
\end{aligned}
$$

\section{Dielectric Material}

In general, high dielectric materials will give absorption curves with values that are much less than unity and have severe fluctuations in magnitude over the frequency range. For high dielectric materials small changes in thickness or in the radius of the shell structure will cause drastic changes in the shape of the absorption curves. This effect is illustrated for a change in radius in figures 2 and 3, where a dielectric constant of 100 has been used. The radius of the spheres has been changed by a factor of two. The scattering coefficient for the same material is shown in figure 4 . The numbers shown in parentheses are $\left(K_{e}^{\prime}, K_{e}^{\prime \prime}\right)\left(K_{m}^{\prime}, K_{m}^{\prime \prime}\right)$, respectively.

\section{Magnetic Material}

The magnetic properties have been investigated on the basis of variation of the permeability $\left(K_{m}^{\prime}\right)$ between 1 and 100 (see fig. 5) and a magnetic loss factor $\left(K_{m}^{\prime \prime}\right)$ between 0.1 and 100 (see fig. 6). The above data is based on a dielectric loss factor $\left(K_{e}^{\prime \prime}\right)$ of 100 and dielectric constant $\left(K_{e}^{\prime}\right)$ of one. Small changes in the general shape of the curves indicate that wide variations in $K_{m}^{\prime}$ and $K_{m}^{\prime \prime}$ have little effect on the uniformity of $C_{a}$. Figures 7 and 8 show the effect on $C_{a}$ for variations of conductivity between $10^{-4}$ and $10^{4} \mathrm{mhos} / \mathrm{m}$ and a permeability of 100 , using a loss tangent of one. The most uniform curve for $C_{a}$ occurs at $(1,1)(100,100)$, indicating that a low conductivity $(\sigma=1 \mathrm{mho} / \mathrm{m})$ is desirable in this combination.

\section{High Conductivity Materials}

The high conductivity case $\left(\sigma>10^{4} \mathrm{mhos} / \mathrm{m}\right)$ occurs whenever the skin depth is an order of magnitude smaller than the shell thickness, so that the fields are damped before reaching the inner sphere. For this case the field coefficients are, from (A14),

$$
a_{n}^{r}=-\frac{j_{n}\left(\xi_{2}\right)}{h_{n}^{(2)}\left(\xi_{2}\right)} \frac{\alpha_{n}^{(1)}\left(\rho_{2}\right)-Z \gamma_{n}\left(\xi_{2}\right)}{\alpha_{n}^{(1)}\left(\rho_{2}\right)-Z \delta_{n}\left(\xi_{2}\right)} .
$$




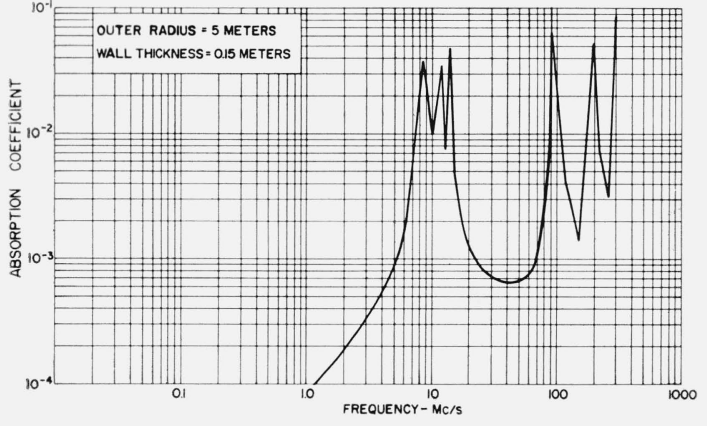

Figure 2. Absorption coefficient versus frequency in $\mathrm{Mc} / \mathrm{s}$ for material with high dielectric constant $(100,0.1)(1,0)$. $a_{2}=5 \mathrm{~m}$.

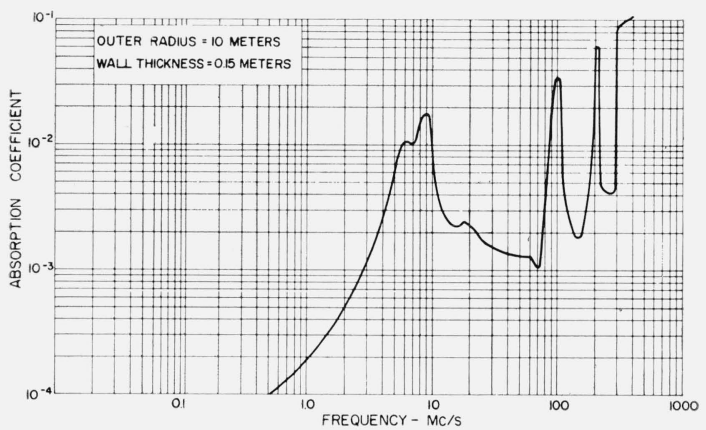

Figure 3. Absorption coefficient versus frequency in $\mathrm{Mc} / \mathrm{s}$ for material with high dielectric constant $(100,0.1)(1,0)$. $a_{2}=10 \mathrm{~m}$.

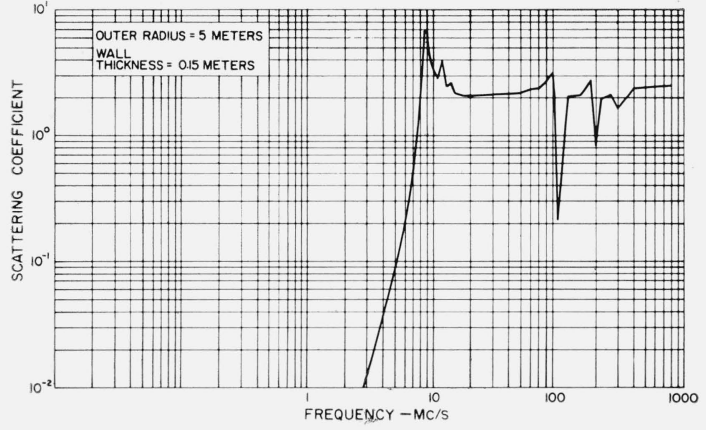

Figure 4. Scattering coefficient versus frequency in $\mathrm{Mc} / \mathrm{s}$ for material with high dielectric constant $(100,0.1)(1,0)$.

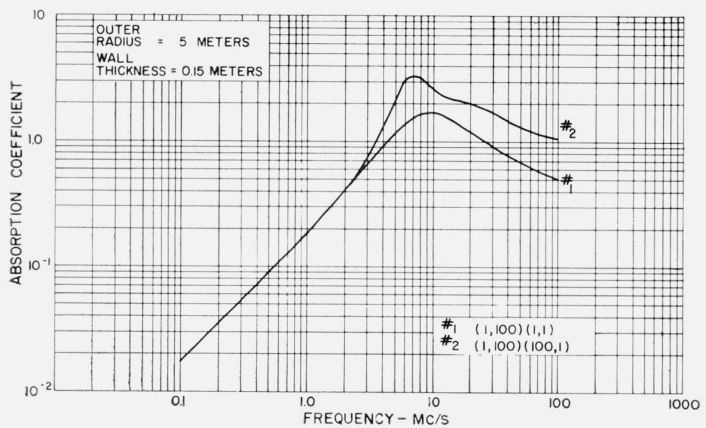

Frgure 5. Absorption coefficient versus frequency in $\mathrm{Mc} / \mathrm{s}$ for variations in permeability between 1 and 100 .

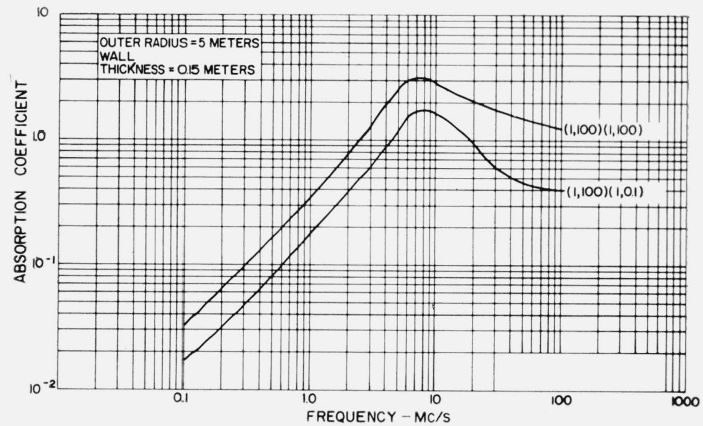

Figure 6. Absorption coefficient versus frequency in $\mathrm{Mc} / \mathrm{s}$ for variations between 0.1 and 100 in the magnetic loss factor.

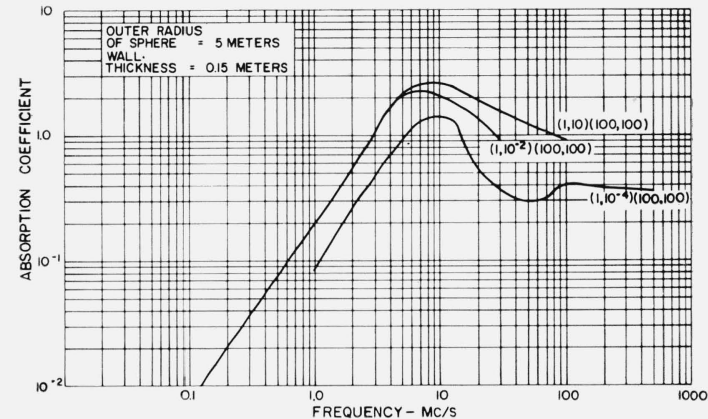

Figure 7. Absorption coefficient versus frequency in $\mathrm{Mc} / \mathrm{s}$. Variations of conductivity from $10^{-1}$ to $10^{-4} \mathrm{mho} / \mathrm{m}$ for high permeability material. $\mu=100$

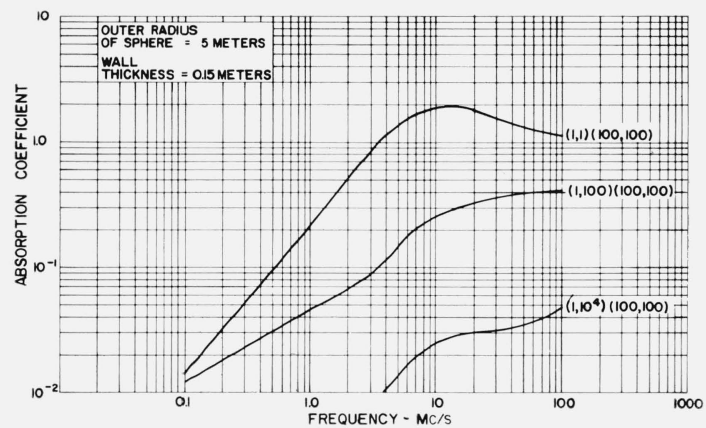

FiguRe 8. Absorption coefficient versus frequency in $\mathrm{Mc} / \mathrm{s}$. Variation of conductivity from 1 to $10^{4} \mathrm{mho} / \mathrm{m}$ for high permeability material. $\mu=100$.

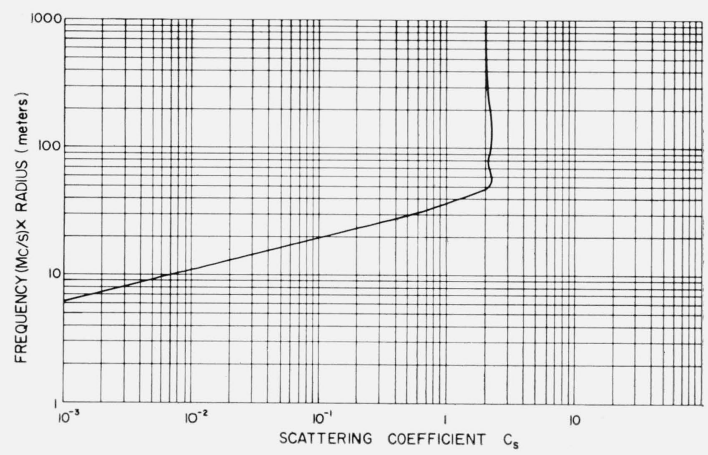

FIgURE 9. Scattering coefficient for conductive materials versus megacycle-meter product. 
Using this, the scattering and absorption coefficients were computed for various material parameters. The resulting data has the following form:

(1) The scattering coefficient is independent of the material parameters and is a function only of the product $\mathrm{Fa}_{2}$ (see fig. 9). For $\mathrm{Fa}_{2}$ less than $20 \mathrm{Mc} / \mathrm{s}$-meter, the scattering coefficient varies as the fourth power of the product $\mathrm{Fa}_{2}$. This is Rayleigh scattering and corresponds to a wavelength greater than 10 times the radius. For $F a_{2}$ greater than $50 \mathrm{Mc} / \mathrm{s}-m e t e r$, the scattering coefficient is approximately two.

(2) The absorption coefficient varies as

$$
C_{a}=\left[\frac{\sqrt{K_{m}^{\prime 2}+K_{m}^{\prime \prime 2}}+K_{m}^{\prime \prime}}{\sigma}\right]^{1 / 2} f_{a}\left(F, a_{2}\right)
$$

where $f_{a}\left(F, a_{2}\right)$ is shown in figure 10 for radii of $1,5,10$, and $20 \mathrm{~m}$. This factor has only a slight dependence on the radius and may be approximated by

$$
f_{a}\left(F, a_{2}\right) \approx \sqrt{F \times 10^{-3}} .
$$

See figure 11 for data on high conductivity materials $\left(\sigma=10^{4}\right.$ to $\left.\sigma=10^{8}\right)$.

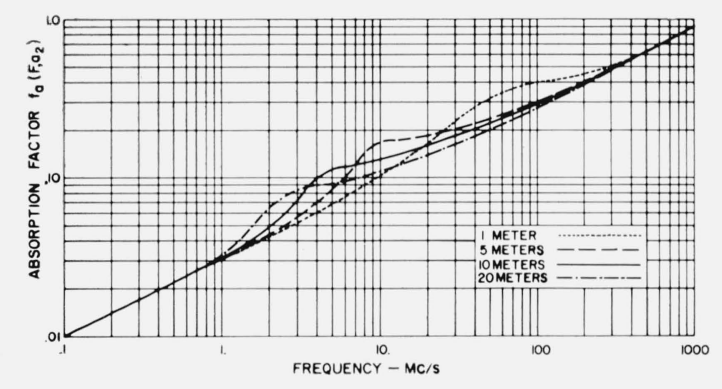

Figure 10. Absorption factor versus frequency in $\mathrm{Mc} / \mathrm{s}$ for conductive materials.

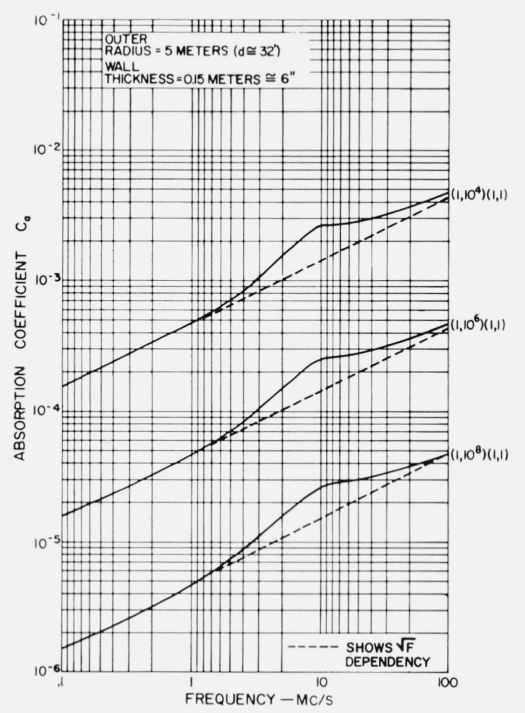

FiguRe 11. Absorption coefficient versus frequency for material conductivity variations between $10^{4}$ and $10^{3} \mathrm{mho} / \mathrm{m}$.

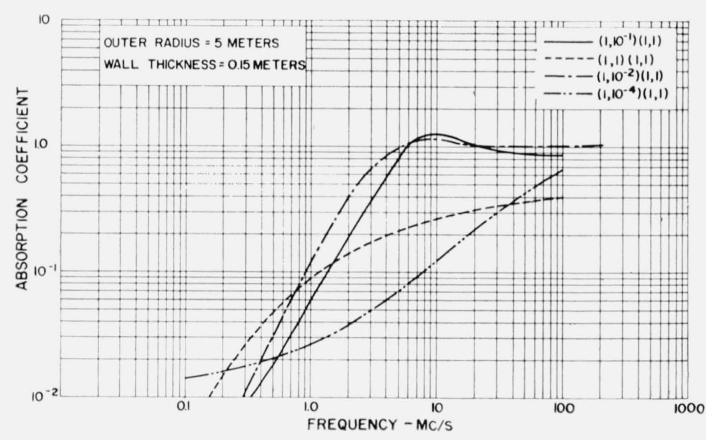

Figure 12. Absorption coefficient versus frequency in $\mathrm{Mc} / \mathrm{s}$ for material conductivity variations between 0.1 and $10^{-4}$ $m h o / m$.

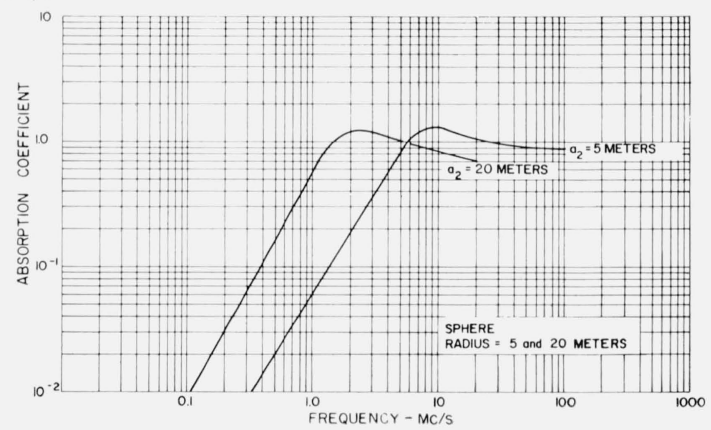

Figure 13. Absorption coefficient versus frequency in $\mathrm{Mc} / \mathrm{s}$ for material of $0.1 \mathrm{mho} / \mathrm{m}$ conductivity. $a_{2}=5 \mathrm{~m}, 20 \mathrm{~m}$.

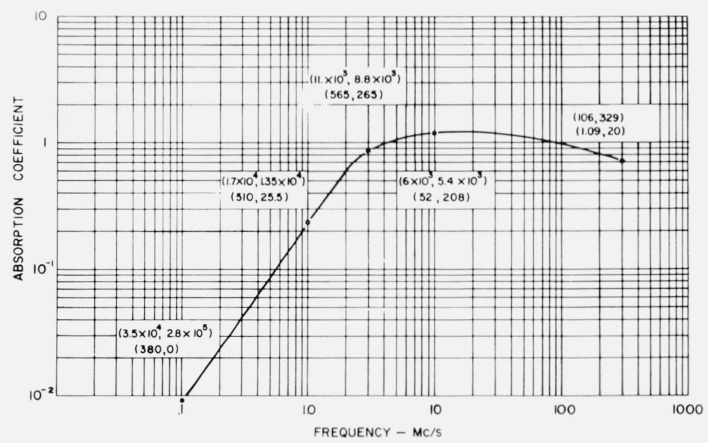

Figure 14. Absorption coefficient for ferramic. 


\section{Conductors}

The approximations made in the high conductivity case are no longer valid for materials less than $10,000 \mathrm{mhos} / \mathrm{m}$. For calculation of $C_{a}$ where $\sigma<10^{4} \mathrm{mhos} / \mathrm{m}$ it has been necessary to use the general equations describing any material. The results of low conductivities (1.0 to $10^{-4} \mathrm{mhos} / \mathrm{m}$ ) are indicated in figure 12 . The most uniform curve of any material examined is given by a material conductivity of $0.01 \mathrm{mhos} / \mathrm{m}$.

Variation of the absorption curve for a change in the radius of the sphere has been investigated. The effect is to shift the maxima downward in frequency as the radius is increased. Figure 13 shows the effect of a radius change from 5 to $20 \mathrm{~m}$ for material $\sigma$ of $0.1 \mathrm{mhos} / \mathrm{m}$. This effect is true for most low to medium conductivity materials.

\section{Variation of Material Parameters With Frequency}

Generally, the variation in $K_{e}^{\prime}, K_{e}^{\prime \prime}, K_{m}^{\prime}, K_{m}^{\prime \prime}$ for most materials is not great over the frequency range of interest in this study. However, the list of materials [Von Hipple, 1954] indicates there is a group of materials whose parameters vary rapidly in the range from 0.1 to 1000 $\mathrm{Mc} / \mathrm{s}$. The listed parameter values for a ferramic were used to determine $C_{a}$ as shown in figure 14. Even with the large changes in $K_{e}$ and $K_{m}$ the curve appears uniform. However, only the data points shown were available and there may be variations due to a lack of data in the intermediate ranges.

\section{Conclusions}

The uniform absorption curves occur for materials whose conductivity is in the region 0.1 to $0.01 \mathrm{mhos} / \mathrm{m}$. Some magnetic materials also give near-uniform absorption curves. Dielectric materials with high dielectric constants and low loss factors are definitely not suitable. Low dielectric constant and high loss factor materials exhibit the characteristics of uniform absorption; however, the low conductivity materials make more suitable building materials.

The absorption coefficient should be as independent as possible of variations in the dimensional properties of the structure. The choice of a material will depend not only on the uniformity of the absorption versus frequency curve, but also the behavior of the absorption coefficient with changes in radius and shell thickness.

\section{Definition of Terms}

$\begin{array}{ll}a_{1} & \text { inner radius } \\ a_{2} & \text { outer radius } \\ a_{n}^{r}, b_{n}^{r} & \text { coefficients of the external field } \\ C_{a} & \text { absorption coefficient } \\ C_{s} & \text { scattering coefficient } \\ E_{0} & \text { magnitude of incident wave } \\ F & \text { frequency (Mc/s) } \\ f_{a}\left(F, a_{2}\right) & \text { absorption factor in }(2) \\ f_{n}^{(l)} & \text { spherical functions defined by }(\mathrm{A} 8) \\ g & \text { relative admittance of material }=\sqrt{K_{e} / K_{m}} \\ h_{n}^{(1)}, h_{n}^{(2)} & \text { first and second spherical Hankel functions } \\ j_{n} & \text { spherical Bessel function of the first kind } \\ k^{e} & \text { propagation constant of material } \\ k_{0} & \text { propagation constant of free space } \\ K_{e} & \text { relative permittivity of material } \\ K_{m} & \text { relative permeability of material } \\ W_{a} & \text { power absorbed (watts) } \\ W_{i} & \text { power density of incident wave }\left(\text { watts } / \mathrm{m}^{2}\right)\end{array}$




$\begin{array}{ll}W_{s} & \text { power scattered (watts) } \\ z_{n}^{(l)} & \text { generalized Bessel function } \\ Z & \text { relative impedance of material }=\sqrt{K_{m} / K_{e}} \\ \alpha_{n}, \gamma_{n}, \delta_{n} & \text { dimensionless functions defined by }(\mathrm{A} 4),(\mathrm{A} 5),(\mathrm{A} 6) \\ \epsilon_{0} & \text { permittivity of free space }=1 / c^{2} \mu_{0} \\ \epsilon^{*} & \text { complex permittivity } \\ \mu_{0} & \text { permeability of free space }=4 \pi \times 10^{-7} \mathrm{H} / \mathrm{m} \\ \mu^{*} & \text { complex permeability } \\ \xi_{s} & \text { dimensionless variable for free space }=k_{0} a_{s} \\ \rho_{s} & \text { dimensionless variable for material }=k a_{s} \\ \sigma & \text { conductivity of material (mhos } / \mathrm{m}) \\ \hat{\imath}_{p} & \text { unit vectors in spherical polar coordinates, } p=1,2,3 \\ \tan \delta_{e}, \tan \delta_{m} & \text { electric and magnetic loss tangent, respectively. }\end{array}$

\section{Appendix A. Derivation of the Mathematical Expressions}

Field coefficients. The following derivation is an application of the method used by Stratton [1941, pp. 563-569] to discuss the diffraction of a plane wave by a single sphere. The same solution may also be obtained by Wait's [1964] general method when specialized to this configuration. The incident plane wave in this paper has a time dependence $(+i \omega t)$ instead "of Stratton's $(-i \omega t)$. The physical configuration is shown in figure 1 .

The fields in the three regions may be expressed in terms of the following spherical vector wave functions, where $x=k r$ and $z_{n}^{(l)}(x)$ is a given spherical Bessel function:

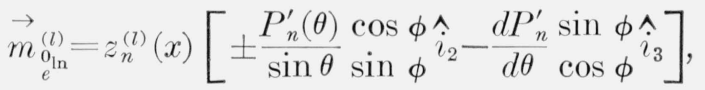

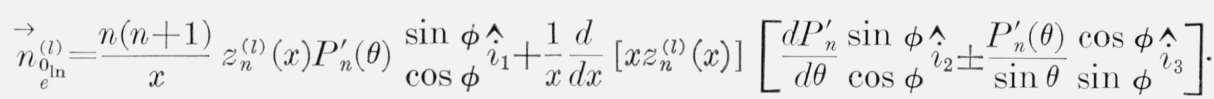

In terms of these vector wave functions and the field coefficients, the fields in the three regions are:

a. Inner region (free space)

where

$$
\begin{aligned}
& \vec{E}=E_{0} e^{i \omega t} \sum_{n=1}^{\infty}(-i)^{n} \frac{2 n+1}{n(n+1)}\left[a_{n}^{t} \vec{m}_{0 \ln }^{(1)}+i b_{n}^{t} \vec{n}_{e \ln }^{(1)}\right] \\
& \vec{H}=\sqrt{\frac{\epsilon_{0}}{\mu_{0}}} E_{0} e^{i \omega t} \sum_{n=1}^{\infty}(-i)^{n} \frac{2 n+1}{n(n+1)}\left[b_{n}^{t} \vec{m}_{e \ln }^{(1)}-i \vec{a}_{n}^{t} \vec{n}_{0 \ln }^{(1)}\right]
\end{aligned}
$$

$$
z_{n}^{(1)}=j_{n} \quad k=k_{0}
$$

b. Middle region (arbitrary material)

$$
\begin{aligned}
& \vec{E}=E_{0} e^{i \omega t} \sum_{n=1}^{\infty}(-i)^{n} \frac{2 n+1}{n(n+1)}\left[\left(a_{n}^{(2)} \vec{m}_{0 \ln }^{(2)}+a_{n}^{(3)} \vec{m}_{0 \ln }^{(3)}\right)+i\left(b_{n}^{(2)} \vec{n}_{e \ln }^{(2)}+b_{n}^{(3)} \vec{n}_{e}^{(3)}\right)\right] \\
& \vec{H}=\sqrt{\frac{\epsilon_{0}}{\mu_{0}}} E_{0} e^{i \omega t} \sum_{n=1}^{\infty}(-i)^{n} \frac{2 n+1}{n(n+1)}\left[\left(b_{\mathrm{n}}^{(2)} \vec{m}_{e \ln }^{(2)}+b_{n}^{(3)} \vec{m}_{e \ln }^{(3)}\right)-i\left(a_{n}^{(2)} \vec{n}_{0 \ln }^{(2)}+a_{n}^{(3)} \vec{n}_{0 \ln }^{(3)}\right)\right]
\end{aligned}
$$

where

$$
z_{n}^{(2)}=h_{n}^{(1)}, z_{n}^{(3)}=h_{n}^{(2)}, k=k_{0} \sqrt{K_{e} K_{m}} .
$$

c. Outer region (free space)

$$
\vec{E}=E_{0} e^{i \omega t} \sum_{n=1}^{\infty}(-i)^{n} \frac{2 n+1}{n(n+1)}\left[\left(\vec{m}_{0 \ln }^{(1)}+a_{n}^{r} \vec{m}_{0 \ln }^{(4)}\right)+i\left(\vec{n}_{e \ln }^{(1)}+b_{n}^{r_{n}\left(\frac{(4)}{e l n}\right)}\right)\right]
$$




$$
\vec{H}=\sqrt{\frac{\epsilon_{0}}{\mu_{0}}} E_{0} e^{i \omega t} \sum_{n=1}^{\infty}(-i)^{n} \frac{2 n+1}{n(n+1)}\left[\left(\vec{m}_{e \ln }^{(1)}+b_{n}^{r} \vec{m}_{e \ln }^{(4)}\right)-i \overrightarrow{\left.\left(n_{0 \ln }^{(1)}+a_{n}^{r} \vec{n}_{0 \ln }^{(4)}\right)\right]}\right.
$$

where

$$
z_{n}^{(1)}=j_{n}, z_{n}^{(4)}=h_{n}^{(2)}, k=k_{0} .
$$

The fields must satisfy the boundary conditions:

$$
\hat{i_{1}} \times\left(\vec{E}^{\prime}-\vec{E}^{\prime \prime}\right)=0 \quad \hat{i_{1}} \times\left(\vec{H}^{\prime}-\vec{H}^{\prime \prime}\right)=0
$$

where primes and double primes refer to two adjacent regions.

Denoting $\xi_{m}=k_{0} \omega_{m}$ and $\rho_{m}=k a_{m}=\xi_{m} \sqrt{K_{e} K_{m}}$, the following relations may be defined:

$$
\begin{gathered}
\frac{d}{d \rho_{m}}\left[\rho_{m} h_{n}^{(l)}\left(\rho_{m}\right)\right]=\rho_{m} h_{n}^{(l)}\left(\rho_{m}\right) \alpha_{n}^{(l)}\left(\rho_{m}\right) ; \alpha_{n}^{(l)}\left(\rho_{m}\right)=\frac{h_{n-1}^{(l)}\left(\rho_{m}\right)}{h_{n}^{(l)}\left(\rho_{m}\right)}-\frac{n}{\rho_{m}} \\
\frac{d}{d \xi_{m}}\left[\xi_{m} j_{n}\left(\xi_{m}\right)\right]=\xi_{m} j_{n}\left(\xi_{m}\right) \gamma_{n}\left(\xi_{m}\right) ; \gamma_{n}\left(\xi_{m}\right)=\frac{j_{n-1}\left(\xi_{m}\right)}{j_{n}\left(\xi_{m}\right)}-\frac{n}{\xi_{m}} \\
\frac{d}{d \xi_{m}}\left[\xi_{m} h_{n}^{(2)}\left(\xi_{m}\right)\right]=\xi_{m} h_{n}^{(2)}\left(\xi_{m}\right) \delta_{n}(\xi) ; \delta_{n}\left(\xi_{m}\right)=\frac{h_{n-1}^{(2)}\left(\xi_{m}\right)}{h_{n}^{(2)}\left(\xi_{m}\right)}-\frac{n}{\xi_{m}}
\end{gathered}
$$

'The field coefficients, determined by substituting the fields into boundary equations, may be written in matrix form as:

$$
\left[\begin{array}{cccc}
j_{n}\left(\xi_{1}\right) & -h_{n}^{(1)}\left(\rho_{1}\right) & -h_{n}^{(2)}\left(\rho_{1}\right) & 0 \\
j_{n}\left(\xi_{1}\right) \gamma_{n}\left(\xi_{1}\right) & -h_{n}^{(1)}\left(\rho_{1}\right) g \alpha_{n}^{(1)}\left(\rho_{1}\right) & -h_{n}^{(2)}\left(\rho_{1}\right) g \alpha_{n}^{(2)}\left(\rho_{1}\right) & 0 \\
0 & h_{n}^{(1)}\left(\rho_{2}\right) & h_{n}^{(2)}\left(\rho_{2}\right) & -h_{n}^{(2)}\left(\xi_{2}\right) \\
0 & h_{n}^{(1)}\left(\rho_{2}\right) g \alpha_{n}^{(1)}\left(\rho_{2}\right) & h_{n}^{(2)}\left(\rho_{2}\right) g \alpha_{n}^{(2)}\left(\rho_{2}\right) & -h_{n}^{(2)}\left(\xi_{2}\right) \delta_{n}\left(\xi_{2}\right)
\end{array}\right]\left[\begin{array}{c}
a_{n}^{t} \\
a_{n}^{(2)} \\
a_{n}^{(3)} \\
a_{n}^{r}
\end{array}\right]=\left[\begin{array}{c}
0 \\
0 \\
-j_{n}\left(\xi_{2}\right) \\
-j_{n}\left(\xi_{2}\right) \gamma_{n}\left(\xi_{2}\right)
\end{array}\right] .
$$

Solving for $a_{n}^{r}$, (1) is obtained. The equations involving the $b_{n}$ 's are identical to those above except the relative admittance $g=\sqrt{K_{e} / K_{m}}$ is replaced by the relative impedance $Z=$ $\sqrt{K_{m} / K_{e}}$

For calculational purposes the following substitutions were found to be useful:

$$
\begin{gathered}
h_{n}^{(1)}(\rho)=\frac{e^{i \rho}}{i \rho} f_{n}^{(1)}(\rho) ; \quad h_{n}^{(2)}(\rho)=\frac{e^{-i \rho}}{-i \rho} f_{n}^{(2)}(\rho) ; \\
\Phi_{n}^{(l)}\left(\rho_{m}\right)=f_{n}^{(l)}\left(\rho_{m}\right)\left[\alpha_{n}^{(l)}\left(\rho_{m}\right)-Z \gamma_{n}\left(\xi_{m}\right)\right]=f_{n-1}^{(l)}\left(\rho_{m}\right)-f_{n}^{(l)}\left(\rho_{m}\right)\left[Z \gamma_{n}\left(\xi_{m}\right)-\frac{n}{\rho_{m}}\right] ; \\
\Lambda_{n}^{(l)}\left(\rho_{2}\right)=f_{n}^{(l)}\left(\rho_{2}\right)\left[Z \gamma_{n}\left(\xi_{2}\right)-Z \delta_{n}\left(\xi_{2}\right)\right] ; \\
R=e^{i\left(\rho_{2}-\rho_{1}\right)} \Phi_{n}^{(2)}\left(\rho_{1}\right) \Phi_{n}^{(1)}\left(\rho_{2}\right)-e^{-i\left(\rho_{2}-\rho_{1}\right)} \Phi_{n}^{(1)}\left(\rho_{1}\right) \Phi_{n}^{(2)}\left(\rho_{2}\right) ; \\
T=e^{i\left(\rho_{2}-\rho_{1}\right)} \Phi_{n}^{(2)}\left(\rho_{1}\right) \Lambda_{n}^{(1)}\left(\rho_{2}\right)-e^{-i\left(\rho_{2}-\rho_{1}\right)} \Phi_{n}^{(1)}\left(\rho_{1}\right) \Lambda_{n}^{(2)}\left(\rho_{2}\right) .
\end{gathered}
$$

Then the reflected field coefficient is given by

$$
a_{n}^{r}=-\frac{j_{n}\left(\xi_{2}\right)}{h_{n}^{(2)}\left(\xi_{2}\right)} \frac{R}{T+R}
$$


For Im $\left(\rho_{2}-\rho_{1}\right)<<-1$, the second terms in (A11) and (A12) are negligible compared to the first terms and the expression for the field coefficient becomes

$$
a_{n}^{r}=-\frac{j_{n}\left(\xi_{2}\right)}{h_{n}^{(2)}\left(\xi_{2}\right)} \frac{\alpha_{n}^{(1)}\left(\rho_{2}\right)-Z \gamma_{n}\left(\xi_{2}\right)}{\alpha_{n}^{(1)}\left(\rho_{2}\right)-Z \delta_{n}\left(\xi_{2}\right)}
$$

This is referred to as the high conductivity case and for a shell thickness greater than about 1 in. is valid for a conductivity greater than $10^{4} \mathrm{mhos} / \mathrm{m}$.

$$
W_{a}=-\frac{\pi E_{0}^{2}}{k_{0}^{2}} \sqrt{\frac{\epsilon_{0}}{\mu_{0}}} \sum_{n=1}^{\infty}(2 n+1)\left(\left|a_{n}^{r}\right|^{2}+\left|b_{n}^{r}\right|^{2}+\operatorname{Re} \boldsymbol{a}_{n}^{r}+\operatorname{Re} b_{n}^{r}\right) \mathrm{W} .
$$

The power density of the incident wave is

$$
W_{i}=\frac{1}{2} E_{0}^{2} \sqrt{\frac{\epsilon_{0}}{\mu_{0}}} \mathrm{~W} / \mathrm{m}^{2} .
$$

Defining the scattering (or absorption) coefficient as the ratio of the power scattered (or absorbed) to the power density of the incident wave times the geometrical cross section, i.e.,

$$
C_{s}=\frac{W_{s}}{\pi a_{2}^{2} W_{i}} \quad C_{a}=\frac{W_{a}}{\pi a_{2}^{2} W_{i}}
$$

The dimensionless coefficients become

$$
\begin{gathered}
C_{s}=\frac{2}{\xi_{2}^{2}} \sum_{n=1}^{\infty}(2 n+1)\left(\left|a_{n}^{r}\right|^{2}+\left|b_{n}^{r}\right|^{2}\right) \\
C_{a}=-\frac{2}{\xi_{2}^{2}} \sum_{n=1}^{\infty}(2 n+1)\left(\left|a_{n}^{r}\right|^{2}+\left|b_{n}^{r}\right|^{2}+\operatorname{Re} a_{n}^{r}+\operatorname{Re} b_{n}^{r}\right) .
\end{gathered}
$$

Mathematical functions. The recursion for all spherical Bessel functions, including $f_{n}^{(l)}$, is

$$
z_{n}(x)=\frac{2 n-1}{x} z_{n-1}(x)-z_{n-2}(x) .
$$

The functions have the initial values

$$
\begin{aligned}
& h_{-1}^{(2)}(\xi)=\frac{\cos \xi}{\xi}-i \frac{\sin \xi}{\xi} ; j_{-1}(\xi)=\frac{\cos \xi}{\xi} \\
& h_{0}^{(2)}(\xi)=\frac{\sin \xi}{\xi}+i \frac{\cos \xi}{\xi} ; j_{0}(\xi)=\frac{\sin \xi}{\xi} \\
& f_{-1}^{(1)}(\rho)=i \quad f_{0}^{(1)}(\rho)=1 \quad f_{-1}^{(2)}(\rho)=i \quad f_{0}^{(2)}(\rho)=1 .
\end{aligned}
$$

Scattering and absorption coefficients. The scattering and absorption coefficients can be expressed in terms of the field coefficients by integrating the radial component of the Poynting vector over a sphere of radius $R$ much larger than the outer radius $a_{2}$.

Separating the fields in (A1) and (A2) into incident fields, $E_{i}$ and $H_{i}$, containing vector wave functions of superscript 1 and Bessel function $J_{n}$, and outgoing fields, $E_{r}$ and $H_{r}$, containing vector wave functions of superscript 4 and Bessel function $h_{n}$, the radial component of the Poynting vector is

$$
S_{R}^{*}=\frac{1}{2}\left(E_{i \theta} \tilde{H}_{i \phi}-E_{i \phi} \tilde{H}_{i \theta}\right)+\frac{1}{2}\left(E_{r \theta} \tilde{H}_{r \phi}-E_{r \phi} \tilde{H}_{r \theta}\right)+\frac{1}{2}\left(E_{i \theta} \tilde{H}_{r \phi}+E_{r \theta} \tilde{H}_{i \phi}-E_{i \phi} H_{r \theta}-E_{r \phi} \tilde{H}_{i \theta}\right) .
$$


The total power scattered, $W_{s}$, and the total power absorbed, $W_{a}$, are

$$
\begin{aligned}
& W_{a}=-\operatorname{Re} \iint S_{R}^{*} R^{2} \sin \theta d \theta d \phi \\
& W_{s}=-\operatorname{Re} \iint \frac{1}{2}\left(E_{r \theta} \tilde{H}_{r \phi}-E_{r \phi} \tilde{H}_{r \theta}\right) R^{2} \sin \theta d \theta d \phi .
\end{aligned}
$$

Using the asymptotic forms for the spherical Bessel functions,

$$
j_{n}(x) \rightarrow \frac{1}{x} \sin \left(x-\frac{n \pi}{2}\right), \quad h_{n}^{(2)}(x) \rightarrow \frac{i}{x} e^{-i\left(x-\frac{n \pi}{2}\right)}
$$

and the relationships,

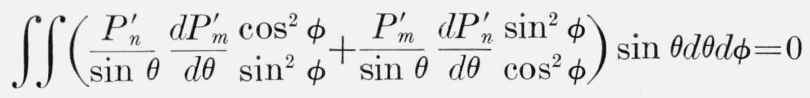

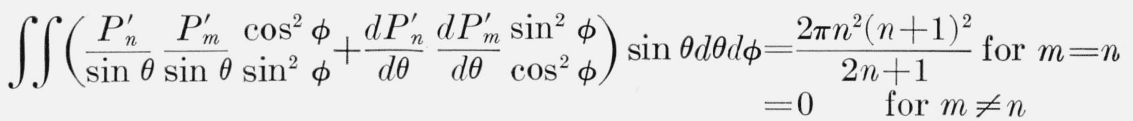

one obtains

$$
W_{s}=\frac{\pi E^{2}}{k_{0}^{2}} \sqrt{\frac{\epsilon_{0}}{\mu_{0}}} \sum_{n=1}^{\infty}(2 n+1)\left(\left|a_{n}^{r}\right|^{2}+\left|b_{n}^{r}\right|^{2}\right) \mathrm{W} .
$$

\section{References}

Salati, O. M. (1962), private communication.

Stratton, J. A. (1941), Electromagnetic Theory, pp. 563-569 (McGraw-Hill Book Co., Inc., New York, N.Y.) Von Hipple, A. (1954), Dielectric Materials and Applications (John Wiley \& Sons, New York, N.Y.).

Wait, J. R. (1962), Electromagnetic Waves in Stratified Media, ch. V (Pergamon Press, Oxford, England).

Wait, J. R. (1964), Electromagnetic scattering from a radially inhomogeneous sphere, Appl. Sci. Res. Sec. B, 10, 567 .

(Paper 68B10-414) 